\title{
Utility of Combining Epworth Sleepiness Scale, STOP- BANG and Perioperative Sleep Apnea Prediction Score for Predicting Absence of Obstructive Sleep Apnea
}

\author{
${ }^{1}$ Mayank Saxena, ${ }^{2}$ Dipti Gothi, ${ }^{3}$ Rambabu Sah, ${ }^{4}$ Umesh C Ojha, ${ }^{5}$ Tanushree Gahlot
}

\begin{abstract}
Aims and objective: To correlate and predict obstructive sleep apnea with the help of 3 sleep questionnaire-perioperative sleep apnea prediction score (PSAP), STOP-BANG, Epworth sleepiness scale score (ESS).

Materials and methods: Prospective study of 69 patients were subjected to 3 sleep questionnaire (PSAP, STOP-BANG, and ESS), then they all were subjected to polysomnography. Obstructive sleep apnea (OSA) was defined for AHI>15, and all these three questionnaires final score was correlated with apnea-hypopnea index ( $\mathrm{AHI})$, and their utility in predicting OSA was calculated (separately as well as when three questionnaire score taken together) using receiver operating characteristic (ROC) curves.
\end{abstract}

Results: Total 69 patients with the mean age of $50.29 \pm 11.37$ years, and male: female of 2:1 and mean body mass index (BMI) of $31.27 \pm 7.253 \mathrm{~kg} / \mathrm{m}^{2}$, mean AHI was calculated to be 19.63 \pm 22.49 and it significantly correlated with PSAP $4.52 \pm 1.596$ $(r=0.376, p=0.001)$, STOP-BANG $4.493 \pm 1.711(r=0.303$, $p=0.011)$, ESS $13.00 \pm 6.782(r=0.395, p=0.001)$. PSAP (area under curve $(A \cup C)=0.761$ ) had a better prediction for OSA than STOP-BANG (AUC $=0.697$ ) and ESS (AUC $=0.669$ ) according to Receiver operating curve. Cut off values for diagnosis of OSA from our data was calculated to be 4 for PSAP (sensitivity: 81\% specificity: $59 \%$ ), 4 for STOP-BANG (sensitivity: $65.4 \%$, specificity: $60 \%$ ) and 11 for ESS (sensitivity: $73.1 \%$; specificity: $48.8 \%$ ). Based on these cut off value 18 patients were found to be having all questionnaire positive of which 11 had OSA $(\mathrm{AHI}>15)$ and rest seven non-OSA. A total of 11 patients were all questionnaire negative of which 10 were non-OSA $(\mathrm{AHI}<15)$. So when three questionnaires were combined to predict OSA their sensitivity was $91.66 \%$ and specificity was $58.8 \%$, a positive predictive value of $61 \%$ and negative predictive value of $90.11 \%$.

Conclusion: Perioperative sleep apnea prediction score (PSAP), STOP-BANG, ESS when taken together can be very helpful in screening for OSA and if all scores are negative it virtually rules out OSA.

\footnotetext{
${ }^{1,5}$ Senior Resident, ${ }^{2}$ Professor, ${ }^{3}$ Medical Officer, ${ }^{4}$ Senior Specialist and Associate Professor

${ }^{1-4}$ Department of Pulmonary Medicine, Employee State Insurance Post Graduate Institute of Medical Sciences and Research, Basaidarapur, New Delhi, India

${ }^{5}$ Department of Pulmonary Medicine, Lady Harding Medical College, New Delhi, India

Corresponding Author: Dipti Gothi, Professor, Department of Pulmonary Medicine, Employee State Insurance Post Graduate Institute of Medical Sciences and Research, Basaidarapur, New Delhi, India, e-mail: diptigothi@gmail.com
}

Keywords: Epworth sleepiness scale, Obstructive sleep apnea, Perioperative sleep apnea prediction score, Prediction, STOP-BANG.

How to cite this article: Saxena M, Gothi D, Sah R, Ojha UC, Gahlot T. Utility of Combining Epworth Sleepiness Scale, Stop-Bang and Perioperative Sleep Apnea Prediction Score for Predicting Absence of Obstructive Sleep Apnea. Indian Sleep Med 2018;13(4):62-66.

Source of support: Nil

Conflict of interest: None

\section{INTRODUCTION}

Obstructive sleep apnea patients are subjected routinely to the sleep questionnaire during primary screening and evaluation., for example, epworth sleepiness scale (ESS), PSAP, STOP-BANG are points based, easy to administer and can be subjected to outpatients settings. However, the utility of sleep-questionnaire is limited as their validation studies were conducted on the different population that's the reason why the sensitivity and specificity of these tests varies a lot. ${ }^{1}$ As per previous literature for predicting moderate to severe OSA the (range of sensitivity/range of specificity) of ESS (39.5 to $75.71 \% / 48.15$ to $71.4 \%$ ), ${ }^{1,2}$ STOP-BANG (87.7 to $97.7 \% / 3.7$ to $43.3 \%),{ }^{1,2}$ PSAP $(67 \%$ to $97.1 \% / 17$ to $77 \%)^{3,4}$ varies widely with study to study and no clear-cut validation of questionnaire-based predictability is available yet. Each of these questionnaires has its merit and demerit viz. ESS is claimed to have a high prediction of excessive daytime sleepiness. ${ }^{5}$ STOPBANG has high sensitivity thus high screening capability, but they lack specificity so higher chances of false positive detection. ${ }^{6,7}$ The PSAP score uses upper airway elements such as high modified Mallampati class ${ }^{8}$ and reduced thyromental distance and includes type 2 diabetes. ${ }^{4}$ Though sleep questionnaire is a very useful tool for the evaluation and predicting OSA in suspected patients, however, there is no guideline which questionnaire to recommend when and all these sleep questionnaires lacks individually on some aspects, and their predictability varies widely. All of the questionnaires can predict the OSA, but their performance in ruling out OSA is still not much looked upon. To overcome these fallacies, in this 
study we have calculated the cut-off and performance of these questionnaires in our study population and have evaluated if the prediction of OSA/ruling out OSA (true positive and true negative) improves when scores of all the three questionnaires are taken together.

\section{METHODOLOGY}

\section{Study design}

It was a retrospective observational study done in the Department of Respiratory Medicine, Employee State Insurance Post Graduate Institute of Medical Sciences and Research (ESI PGIMSR) and ESI hospital Basaidarapur New Delhi with an aim to correlate and predict obstructive sleep apnea with the help of 3 sleep questionnairePSAP, STOP-BANG, ESS score. After a thorough clinical examination, all study patients were interviewed for all the three, and their polysomnography was considered gold standard in diagnosing sleep disordered breathing.

\section{Study Population}

The study was conducted on patients who were attending the outpatient department (OPD) and were having a high suspicion for OSA. Approval was taken from an ethical committee for evaluating the data. The patients with age $>18$ years with the history suggestive of sleepdisordered breathing were included. Physical examination and anthropometric features like age, BMI, neck circumference, waist circumference were noted. All the 69 suspected patient had been subjected to three questionnaires viz. ESS, STOP-BANG and PSAP. Their (polysomnography) was reviewed for correlation.

\section{Questionnaire}

Epworth sleepiness scale score (ESS): A self administered questionnaire that asks subjects to rate their chances of falling asleep, which is scored in a scale from 0-3 (0 = would never dose, 1 = slight chance of dozing, $2=$ moderate chance of dozing, and 3 = high chance of dozing) in eight situations that are routinely encountered in daily life. The total ESS score is the sum of 8-items scores and can range between 0 and 24 . The higher the score, the higher the person's level of daytime sleepiness as follows: normal, 0 to 10; and excessive daytime sleepiness, 11 to $24 .^{5}$ However, in our study population, we have computed the cut off value of ESS in predicting moderate to severe OSA using ROC curve as the sensitivity and specificity has varied widely for earlier cut off values.

STOP-BANG: The STOP-BANG questionnaire is a scoring model consisting of eight easily administered questions starting with the acronym STOP-BANG and is scored based on yes/no answers (score: $1 / 0$ ). Thus, the scores range from a value of 0 to 8 . A score of $\geq 3$ has shown a high sensitivity for detecting OSA. ${ }^{6,7}$ For this study, we have calculated the cut off value for moderate-severe OSA $(\mathrm{AHI}>15)$ in our study population using ROC curves. Perioperative sleep apnea prediction (PSAP): The PSAP score validates 6 of the eight elements of the STOPBANG model but differs in that it uses a high modified Mallampati class, reduced thyromental distance and includes type 2 diabetes. Modified Mallampati class is a validated marker of diagnosis and severity of OSA. ${ }^{8,9} \mathrm{We}$ have calculated the cut off value for moderate to severe OSA for our study population using ROC curves.

\section{Polysomnography}

The diagnostic full night type 1 PSG was performed using computerized Alice 5 polysomnograph system (in institution-based laboratory) with the monitoring of electroencephalogram (EEG), submental and anterior tibial electromyogram (EMG), oxygen saturation, electrocardiogram (ECG), inductance plethysmography of chest wall and abdomen, nasal pressure sensor, and oronasalthermister. The sleep stage and event scoring [as per American Academy of Sleep Medicine (AASM) Manual for the Scoring of Sleep and Associated Events] was done manually by the sleep specialist blinded to the results of the questionnaires. ${ }^{10}$ Total obstructive apnea (OA)/AHI was calculated as the number of obstructive apneas and hypopneas per hour of total sleep time (TST). AHI >15 was taken as moderate to severe OSA

\section{Statistical Analysis}

The collected data were analyzed by statistical package for the social sciences (SPSS) 21, baseline characters were noted, the result of PSG was considered gold standard in the diagnosis of OSA. Correlation of all the three questionnaires with AHI was done individually. Separate cut off values and sensitivity specificity of each questionnaire was calculated by using ROC curves and two by two tables. Performance of all questionnaires taken together in predicting/ruling out OSA was calculated

\section{RESULTS}

A total of 69 patients were evaluated, the demographics are as shown in (Table 1), mean age was $50.29 \pm 11.37$ years, out of which 46 were male and 23 were female. The mean BMI of patients was $31.27 \pm 7.253 \mathrm{Kg} / \mathrm{m}^{2}$. Patients were having mean AHI of $19.62+22$ events/ hour. Patients questionnaire mean \pm SD for PSAP was $4.52 \pm 1.596$, STOP-BANG was $4.49 \pm 1.711$ and ESS was $13.00 \pm 6.782$. On correlation analysis all the three questionnaire PSAP $\left(\mathrm{r}^{2}=0.376 \mathrm{p}=0.001\right)$ STOP-BANG $\left(\mathrm{r}^{2}=\right.$ 


\begin{tabular}{ll}
\hline \multicolumn{2}{c}{ Table 1: Demographics } \\
\hline & Mean \pm SD \\
\hline Age & $50.29 \pm 11.37$ \\
Spo2 (room air) & $96.39 \pm 7.12$ \\
STOP-BANG & $4.493 \pm 1.711$ \\
PSAP & $4.52 \pm 1.596$ \\
ESS & $13.00 \pm 6.782$ \\
Neck circumference $(\mathrm{cm})$ & $39.8 \pm 3.75$ \\
Waist circumference $(\mathrm{cm})$ & $102.94 \pm 24.025$ \\
BMI (Kg/M & $31.27 \pm 7.25$ \\
Predicted FeV1 \%\% & $64.51 \pm 21$. \\
AHI & $19.63 \pm 22.49$ \\
Min Spo2 \% (during PSG) & $74.687 \pm 16.34$ \\
\hline
\end{tabular}

0.303, $\mathrm{p}=0.011)$, ESS $\left(\mathrm{r}^{2}=0.395, \mathrm{p}=0.001\right)$ were found to be positively correlated with AHI (Fig. 1). The ROC was drawn for each of the questionnaire for predicting OSA, and cut off values were derived for a questionnaire which can predict AHI of more than 15 (Fig. 2). The PSAP area under the curve $(\mathrm{AUC})=0.761)$ had a better prediction for OSA than STOP-BANG (AUC $=0.697$ ) and ESS $($ AUC $=0.669)$ according to ROC. Cut-off values for diagnosis of moderate OSA from our data was calculated to be 4 for PSAP (sensitivity: $81 \%$ specificity: $59 \%$ ), 4 for STOP-BANG (sensitivity: $65.4 \%$, specificity: $60 \%$ ) and 11 for ESS (sensitivity: $73.1 \%$; specificity: $48.8 \%$ ) (Table 2).

The questionnaire was marked positive if the score was above the cut-off value and negative if the score below the cut-off value as computed by the ROC curve. When questionnaire scores were taken together. So out of 69 patients, 18 patients were all questionnaire positive and 11 patients were all questionnaire negative. Out of 18 patients who were positive for all questionnaire, 11 patients had moderate to severe OSA $(\mathrm{AHI}>15)$ as diagnosed on PSG, rest seven patients had AHI $<15$. All questionnaire score analyzed together were able to
Table 2. Predictive probability of sleep questionnaire and all questionnaire score taken together

\begin{tabular}{lllll}
\hline $\begin{array}{l}\text { Questionnaire/ } \\
\text { probability }\end{array}$ & $\begin{array}{l}\text { STOP- } \\
\text { BANG }\end{array}$ & PSAP & ESS & All questionnaire \\
\hline Senstivity & $65.4 \%$ & $81 \%$ & $76 \%$ & $91.66 \%$ \\
$\begin{array}{l}\text { Specificity } \\
\text { +ve predictive }\end{array}$ & $60 \%$ & $59 \%$ & $50 \%$ & $58.8 \%$ \\
$\begin{array}{l}\text { value } \\
\text {-ve predictive } \\
\text { value }\end{array}$ & $75 \%$ & $51.2 \%$ & $53.60 \%$ & $61 \%$ \\
\hline
\end{tabular}

successfully rule out OSA in 11 patients out of which 10 patients did not have moderate to severe OSA as confirmed by PSG. All questionnaire score had the sensitivity of $91.66 \%$, specificity of $58.8 \%$, the positive predictive value of $61 \%$ and negative predictive value of $91 \%$ in predicting OSA (Table 2).

\section{DISCUSSION}

This study aims to see the role of three questionnaires viz. STOP-BANG, ESS, PSAP in predicting moderate to severe OSA and effects on predictive probabilities when the score of the three questionnaires are taken together. The study population was mid-elderly age patients who were suspected of OSA, questionnaires were administered in outpatient settings and PSG was considered the gold standard for the diagnosis of OSA. All questionnaires correlated positively with AHI consistent with previous studies. ${ }^{11,12}$ In our research, we have taken moderate to severe OSA viz. AHI >15, as we consider these patients to be primarily targeted for therapeutic approach. As previous studies have shown wide variation in sensitivity and specificity for the questionnaire, so in this study we have computed the cut off values for each questionnaire in predicting OSA in our study population with the help of ROC curve, our results were consistent with the
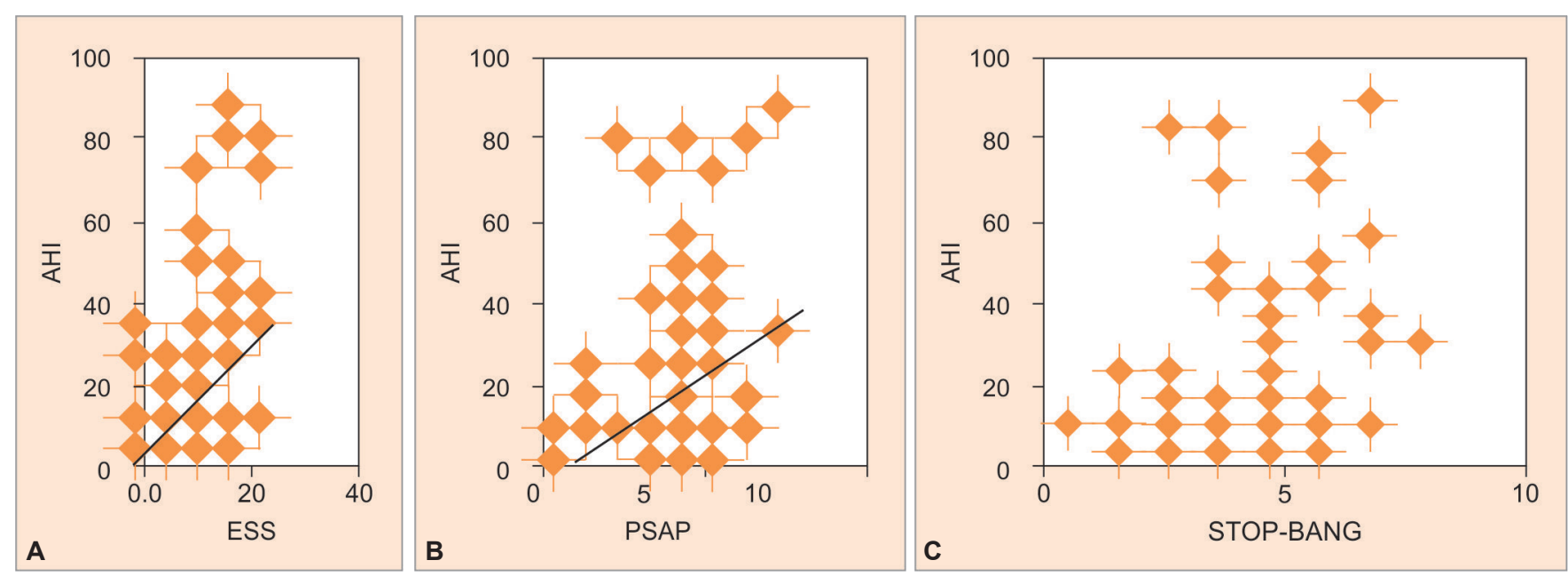

Figs $1 \mathrm{~A}$ to $\mathrm{C}$ : Correlation of $\mathrm{AHI}$ with individual questionnaire (A) ESS with AHI; (B). PSAP with AHI; (C) STOP-BANG with AHI. This figure shows correlation of each questionnaire with AHI separately. PSAP $\left(r^{2}=0.376 p=0.001\right)$ STOP-BANG $\left(r^{2}=0.303, p=0.011\right)$, ESS $\left(r^{2}=0.395, p=0.001\right)$ were found to be positively correlated with AHI. PSAP: Perioperative sleep apnea score, ESS : Epworth sleepiness scale, STOP-BANG questionnaire. AHI: apnea hypopnea index 


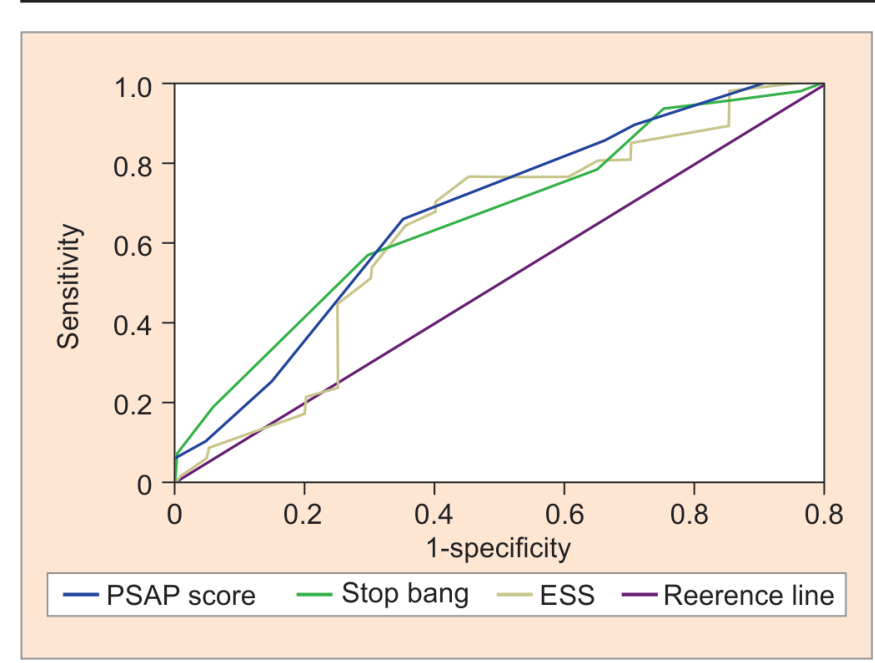

Fig. 2: ROC curve for determining cut off values of questionnaire in predicting OSA. The ROC curve : A graphical plot displaying the ability to distinguish between subjects with and without a disease. Overall accuracy of the ROC curve is expressed as the area under the ROC curve (AUC), which ranges between 0 and 1 . The closer the AUC is to 1, better is the diagnostic accuracy of the test. PSAP (area under curve $(A \cup C)=0.761$ ) had better prediction for OSA than STOP-BANG (AUC=0.697) and ESS (AUC=0.669)

previously designated cut off for the ESS and PSAP i.e. $>11$ and $>4$ respectively. In our study population predictive performance was similar to as available in the previous literature, for the above mentioned cut off in ESS with the sensitivity of $73.1 \%$; specificity of $48.8 \%$ and PSAP of sensitivity $81 \%$ and specificity $59 \%$ were well following quoted values in previous literature. However the STOPBANG was calculated to be having cut off value of $>4$, in previous studies, the cut off value have been ranged from $>3$ to $>5$. American Society of Anesthesiology (ASA) has recommended following scale for STOP-BANG, 0 to 2: no risk for OSA, 3 to 5: intermediate risk, and $>5$ : high risk for OSA, the overall sensitivity of STOP-BANG is low $(65.4 \%)$ with our study compared to earlier studies $(87-91 \%) .{ }^{13}$ The reason can be due to raising the cut off value on the cost of improved specificity and thus less false positive cases as computed on ROC curves in our study. The interesting part of our study was when the result of all questionnaires were computed together the sensitivity improved markedly. To the best of our knowledge, no previous studies have assessed for predictability of OSA with three questionnaire score taken together. Earlier Ulasli et al. advocated the use of ESS and Berlin's questionnaire together as they had slightly better performance when used together than when they are taken alone in predicting OSA. ${ }^{14}$ In our study we found the overall improvement in performance of all three questionnaires taken together compared to each individual questionnaire, the sensitivity was $91.66 \%$, specificity of $58.8 \%$. All sleep questionnaires predicted sleep apnea in18 patients out of which 11 were confirmed to be having moderate-severe sleep apnea on PSG making the positive predictive value of $61 \%$ which is lowered as compared to the individual questionnaire which can be attributed to the high sensitivity of combined questionnaire taken together. When patients who were negative for all the sleep questionnaire were analyzed we could find it was able to rule out sleep apnea in 11 patients out of which 10 patients were proven for not having moderate-severe sleep apnea. The only one patient which had all three questionnaires negative and diagnosed to have sleep apnea positive hadvery severe anxiety and insomnia. He was on 3 antipsychotic drugs, his insomnia was so severe that he did not have day time sleepiness despite having sleep apnea and disturbed nocturnal sleep. So if we exclude psychiatric patients ${ }^{15}$ combination of "all questionnaires" could rule out OSA with nearly 100\% sensitivity. On analysis of previous literature it is found that emphasis of the questionnaire is mainly on detecting the sleep apnea ${ }^{16,17}$ but in our study, we have analyzed whether the questionnaire has any utility in ruling out OSA, i.e. negative predictive value. So in our study we assume that if we subject the patient to the three questionnaire as mentioned, and if all the questionnaires are found to be negative then it virtually rules out OSA. However our study has a limitation that no. of patients were lesser ascompared to previously done studies, so more studies are required with large patient data pool so as to evaluate the effectiveness of impact of multiple questionnaire evaluation in patients suspected of OSA. Still, In our resource limited settingsthis finding can be extremely useful in avoiding unnecessary PSG.

\section{CONCLUSION}

Perioperative sleep apnea prediction score (PSAP), STOP-BANG, ESS, when taken together, have a very high sensitivity in ruling out moderate to severe OSA. If PAPS $<4$, STOP-BANG $<4$, ESS $<11$ (all questionnaire negative) and psychosis are ruled out sleep study for OSA are not required.

\section{REFERENCES}

1. Silva GE, Vana KD, Goodwin JL, Sherrill DL Quan SF. Identification of patients with sleep disordered breathing: comparing the Four-Variable screening tool, STOP, STOPBang, and Epworth Sleepiness Scales. J Clin Sleep Med 2011 Oct 15;7(5):467-472

2. Sayed IHE. Comparison of four sleep questionnaires for screening obstructive sleep apnea Egypt J Chest Dis AdnTuberc 2012 Oct 1;61(4):433-441.

3. Ramachandran SK, Kheterpal S, Consens F, Shanks A, Doherty TM, Morris M, Tremper KK. Derivation and validation of a simple perioperative sleep apnea prediction score. AnesthAnalg 2010 Apr 1;110(4):1007-10015.

4. Sundar E, Chang J, Smetana GW. Perioperative Screening for and Management of Patients with Obstructive Sleep Apnea, JCOM. 2011 Sep;18(9):399-401. 
5. Johns MW. A new method for measuring daytime sleepiness: the Epworth Sleepiness Scale. Sleep1991;14(6):540545

6. Chung F, Yegneswaran B, Liao P, Chung SA, Vairavanathan S, Islam S, Khajehdehi A, Shapiro CM. STOP QuestionnaireA Tool to screen patients for obstructive sleep apnea. Anesthesiology: The Journal of the American Society of Anesthesiologists. 2008 May 1;108(5):812821.

7. Ong TH, Raudha S, Fook-Chong S, Lew N, Hsu AA. Simplifying STOP-BANG: use of a simple questionnaire to screen for OSA in an Asian population. Sleep and Breathing. 2010 Dec 1;14(4):371-376.

8. Ramachandran SK, Josephs LA. A meta-analysis of clinical screening tests for obstructive sleep apnea. Anesthesiology. 2009 Apr;110(4):928-939.

9. Chung F. Screening for Obstructive Sleep Apnea Syndrome in the Preoperative Patients. The Open Anesthesiol J, 2011,5: 7-11

10. ASA criteria for predicting OSA. https://www.sleepassociation.org/sleep-apnea-screening-questionnaire-stop-bang/ as accessed on 10-2-2016.

11. Luo J, Huang R, Zhong X, Xiao Y, Zhou J. Value of STOPBang questionnaire in screening patients with obstructive sleep apnea hypopnea syndrome in sleep disordered breathing clinic. Chinese medical journal. 2014 May 20;127(10):1843-1848.

12. Quaranta VN, Dragonieri S, Carratu P, Falcone VA, Carucci E, Ranieri T, Ventura V, Resta O. A new approach for the assessment of sleepiness and predictivity of obstructive sleep apnea in the drivers : A pilot study. Lung India: official organ of Indian Chest Society. 2016 Jan;33(1):14-19.

13. Chung F, Subramanyam R, Liao P, Sasaki E, Shapiro C, Sun Y. High STOP-Bang score indicates a high probability of obstructive sleep apnoea. British journal of anaesthesia. 2012 Mar 8;108(5):768-775.

14. Ulasli SS, Gunay E, Koyuncu T, Akar O, Halici B, Ulu S, Unlu M. Predictive value of Berlin Questionnaire and Epworth Sleepiness Scale for obstructive sleep apnea in a sleep clinic population. ClinRespir J 2014 Jul;8(3):292-296.

15. Abrishami A, Khajehdehi A, Chung F. A systematic review of screening questionnaires for obstructive sleep apnea. Can J Anaesth. 2010 May;57(5):423-438.

16. Kim B, Lee EM, Chung YS, Kim WS, Lee SA. The utility of three screening questionnaires for obstructive sleep apnea in a sleep clinic setting. Yonsei Med J 2015 May 1;56(3):684-90

17. Ramachandran SK, Josephs LA. A meta-analysis of clinical screening tests for obstructive sleep apnea. Anesthesiology 2009 Apr 1;110(4):928-939. 